\title{
Obtenção e análise dos dados cinéticos da reação de saponificação do Acetato de Etila em um reator Batelada agitado
}

\author{
Suênia Fernandes de Vasconcelos* (Graduanda em Engenharia Química na Universidade Federal de Campina \\ Grande - UFCG); \\ Carla Gabriela Azevedo Misael (Graduanda em Engenharia Química na Universidade Federal de Campina \\ Grande - UFCG); \\ Caroline Vasconcelos Fernandes (Graduanda em Engenharia Química na Universidade Federal de Campina \\ Grande - UFCG); \\ Filipe José Ferreira Chaves (Graduando em Engenharia Química na Universidade Federal de Campina Grande - \\ UFCG); \\ Josiele Souza Batista Santos (Graduanda em Engenharia Química na Universidade Federal de Campina Grande - \\ UFCG); \\ Juan Nicolas Andrade Cavalcante (Graduando em Engenharia Química na Universidade Federal de Campina \\ Grande - UFCG). \\ *Email: sueniaafernandes@gmail.com
}

Resumo:

O Acetato de Etila é um composto orgânico da família dos ésteres com alto poder de solvência, muito utilizado em industrias de tintas e vernizes, mas também pode ser empregado sobre suportes, como filme celulósico, papel, folha de alumínio, PVC, poliéster, polietileno e polipropileno. Quando esse composto é reagido com uma base forte, ele é hidrolisado e essa reação é chamada de saponificação. A reação é complexa e não elementar, com várias reações intermediarias. Sendo assim, faz-se necessário estudar e conhecer os métodos cinéticos, que consistem em análises de correlações baseadas em dados cinéticos, oriundos de procedimentos experimentais. Nesse trabalho aplicou-se 0 método diferencial com o objetivo de encontrar uma ordem de reação que satisfizesse as condições reacionais do processo. Foram usadas três concentrações para o Acetato de Etlia $(0,1 \mathrm{M} ; 0,04 \mathrm{M}$ e $0,08 \mathrm{M}$ ) e os dados coletados em regime transiente. A concentração final da base usada como reagente (Hidróxido de Sódio) pôde ser mensurada a partir de valores de conversão calculados pelo software CEB-Armfield. Com base nesses dados, gráficos da concentração do Hidróxido de Sódio em função do tempo foram plotados e as derivadas das curvas em análise foram calculadas por regressão linear, possibilitando o cálculo da ordem da reação. Entretanto, é importante destacar que a não linearidade dos dados experimentais torna a regressão linear apenas uma aproximação do parâmetro. Quanto à conversão de $\mathrm{NaOH}$, verifica-se que no experimento de concentrações de $0,1 \mathrm{M}$ para o Acetato de Etila a conversão foi maior quando comparado com os outros experimentos. Esse fato ocorre por não existir reagente limitante, fazendo com que os compostos reajam efetivamente, provocando um aumento no valor da conversão.

Palavras-chave:

Acetato de Etila; CEB-Armfield; ordem de reação; conversão 


\section{I ntrodução}

Dentre os diversos problemas de engenharia, tanto de projeto como de processo, a reação química acompanhada de fenômenos de transporte ocupa um lugar de destaque em uma unidade completa de operações unitárias. Pode-se dizer que do projeto ou da operação do reator químico dependerão os projetos e as operações das demais unidades, que os precedem ou que os sucedem, bem como a instrumentação e o sistema de controle. O estudo da reação e do reator é, portanto, de fundamental importância (FROST e PEARSON, 1952).

O Acetato de Etila $\left(\mathrm{CH}_{3} \mathrm{COOC}_{2} \mathrm{H}_{5}\right)$, também conhecido como Etanoato de Etila, é da família dos ésteres e tem um alto poder de solvência, sendo muito utilizado como solvente polar. É um líquido, límpido, incolor e com o odor forte e frutal, de polaridade moderada e instável na presença de bases fortes e ácidos aquosos. Em condições ambientes, possui um ponto médio de ebulição de $77^{\circ} \mathrm{C}$, ponto de fulgor de $-4{ }^{\circ} \mathrm{C}$ e temperatura de autoignição de $426{ }^{\circ} \mathrm{C}$ (CHEMISOL, 2015).

Quando se reage um éster com uma base forte como o hidróxido de sódio, ocorre a hidrólise do éster, sendo essa reação chamada de saponificação. A hidrólise do acetato é uma reação complexa, chamada reação não elementar, que possui uma série de etapas intermediárias de reações elementares que constituem o mecanismo da reação. Sendo assim, quando se estuda a cinética de uma reação, observa-se que a ordem de reação nem sempre coincide com a estequiometria e as equações de taxa precisam ser testadas frente a dados experimentais (HILL e ROOT, 1977).

Para que se conheça a lei de velocidade de determinada reação, realiza-se análises e correlações de dados cinéticos, os métodos cinéticos. Estes, consistem em procedimentos que contrapõe equações de taxa e dados experimentais. Comumente, são empregados seis diferentes tipos de métodos de análise dos dados coletados: o método integral, o diferencial, o de meias-vidas, o das velocidades iniciais, o da regressão linear e o de regressão não-linear. Tais métodos são utilizados de forma que se adequem aos dados cinéticos que se detêm a partir do reator utilizado. Para a reação de saponificação do Acetato de Etila (reação homogênea), é indicado o uso de um reator batelada, também conhecido como reator tanque descontínuo, operando isotermicamente (FOGLER, 2009). Os dados são coletados em regime transiente e a concentração é medida como uma função do tempo. Logo, os métodos integral e diferencial são os mais utilizados para esse tipo de reator.

O método integral de análise é o de uso mais simples. Ele é empregado mais frequentemente quando a ordem da reação é conhecida e se deseja calcular a velocidade específica da reação a diferentes temperaturas, tendo em vista a determinação da energia de ativação. Normalmente, nesse método, supõe-se uma ordem de reação e integra-se a equação diferencial utilizada para modelar o sistema em batelada. Se a suposta ordem de reação for a correta, o gráfico correspondente dos dados de concentração-tempo deve ser razoavelmente linear (LEVENSPIEL, 2000). Comumente, em situações complexas, quando o método integral não se mostra eficaz, o método diferencial é utilizado, aplicando-se diretamente na equação diferencial da velocidade a ser testada. O mesmo permite uma avaliação de todos os termos da equação (FOGLER, 2009).

A reação de saponificação do éster, representada pela Equação 1, ocorre por substituição nucleofílica, no qual o íon hidroxila é adicionado ao grupo acetil, enquanto o íon sódio forma um sal orgânico com o íon acetato, permanecendo ionizado na solução. Assim, quando o Acetato de Etila é saponificado com hidróxido de sódio, ocorre a formação de Acetato de Sódio e Álcool Etílico (SOLOMONS E FRYHLE, 2008).

$$
\mathrm{CH}_{3} \mathrm{COOC}_{2} \mathrm{H}_{5}+\mathrm{NaOH} \rightarrow \mathrm{CH}_{3} \mathrm{COONa}+\mathrm{CH}_{3} \mathrm{CH}_{2} \mathrm{OH}
$$

As Figuras 1 e 2 representam o mecanismo de reação proposto pela literatura para a reação de saponificação do Acetato de Etila com uma base forte. 
Figura 1 - Ataque nucleofílico da hidroxila ao grupo carbonila do éster e eliminação do grupo acetato.

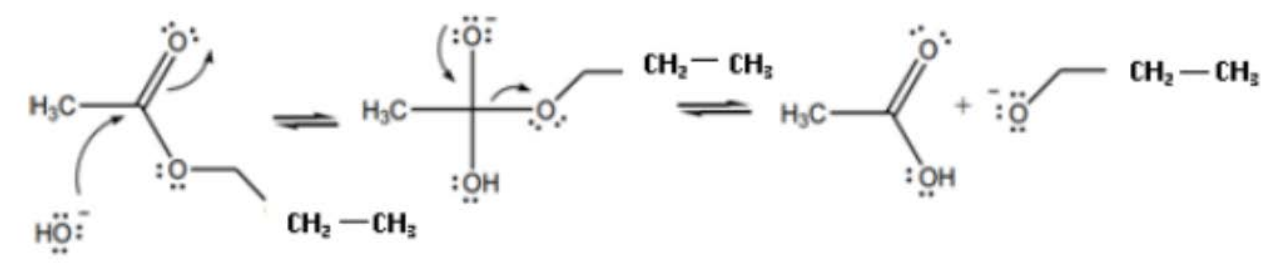

Fonte: Adaptado (SOLOMONS e FRYHLE, 2008).

Figura 2 - Desprotonação do ácido acético em meio básico para a produção do acetato.

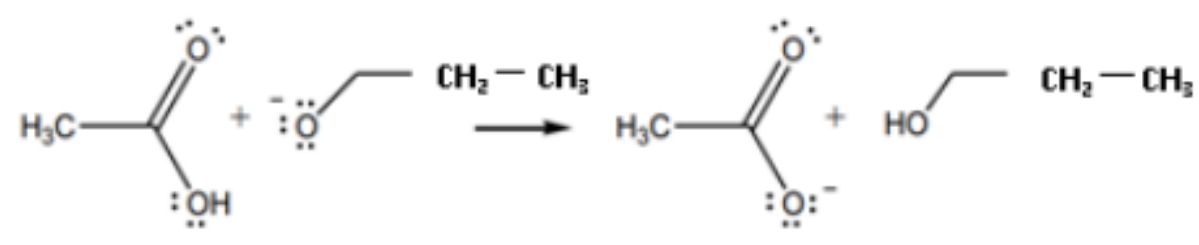

Fonte: Adaptado (SOLOMONS e FRYHLE, 2008).

A reação acima é considerada uma reação de saponificação pelo fato de representar a mesma hidrólise alcalina que ocorre a partir de um ácido graxo para a produção de sabão. O íon carboxilato é muito pouco reativo frente à substituição nucleofílica porque ele é carregado negativamente. Por esse motivo, a hidrólise de um éster promovida por uma base é uma reação essencialmente irreversível. A saponificação do Acetato de Etila é uma reação lenta, bimolecular, na qual a ordem da reação pode variar entre 0 e 2 (KRÜGER et al., 2013).

Um processo químico é idealizado para que se obtenha um produto desejado, contudo, devido à diversidade de matérias-primas, produtos indesejados também são produzidos. Para que exista uma viabilidade econômica ao final do processamento, o produto desejado deve ser predominante, sendo necessária algumas operações unitárias e tratamentos físicos antes e depois do reator. Se um sistema de reações produz uma grande quantidade de produto indesejável, a purificação e a separação subsequentes do produto desejado poderão tornar o processo inteiro economicamente desfavorável. Dessa forma, a seleção de um sistema de reação que opere de maneira mais segura e eficiente pode ser a chave para o sucesso ou o fracasso econômico de uma planta química (FOGLER, 2009).

Nesse contexto, esse trabalho teve como finalidade determinar as ordens de reações das hidrólises do Acetato de Etila com concentrações de 0,1; 0,08 e 0,04 mol.dm ${ }^{-3}$ em meio alcalino (Hidróxido de Sódio) com concentração de $0,1 \mathrm{~mol}^{-\mathrm{dm}^{-3}}$. As bateladas foram realizadas em uma temperatura de $30^{\circ} \mathrm{C}$. Além disso, foi possível verificar a conversão de Hidróxido de Sódio $(\mathrm{NaOH})$ e a produção de Acetato de Sódio.

\section{Metodologia}

Realizou-se o experimento a partir de soluções com concentrações de $0,1 \mathrm{~mol}^{\mathrm{d}} \mathrm{dm}^{-3}$ de hidróxido de sódio e $0,1 \mathrm{~mol}_{\mathrm{dm}}{ }^{-3}$ de Acetato de Etila. Inicialmente, ajustou-se o reator para operar a uma temperatura de reação de $30^{\circ} \mathrm{C}$. Adicionou-se ao reator (Figura 3) $0,5 \mathrm{~L}$ de cada solução e uma pitada de indicador cristal violeta, e ligou-se a agitação e a circulação de água quente na jaqueta. Posteriormente, iniciou-se a reação e coletou-se uma determinada quantidade de dados em um correspondente intervalo de tempo. O experimento também foi realizado variando as concentrações de Acetado de Etila em 0,08 e 0,04 mol.dm ${ }^{-3}$ (Tabela 1 ). 
Figura 3 - Reator utilizado para realização do experimento.

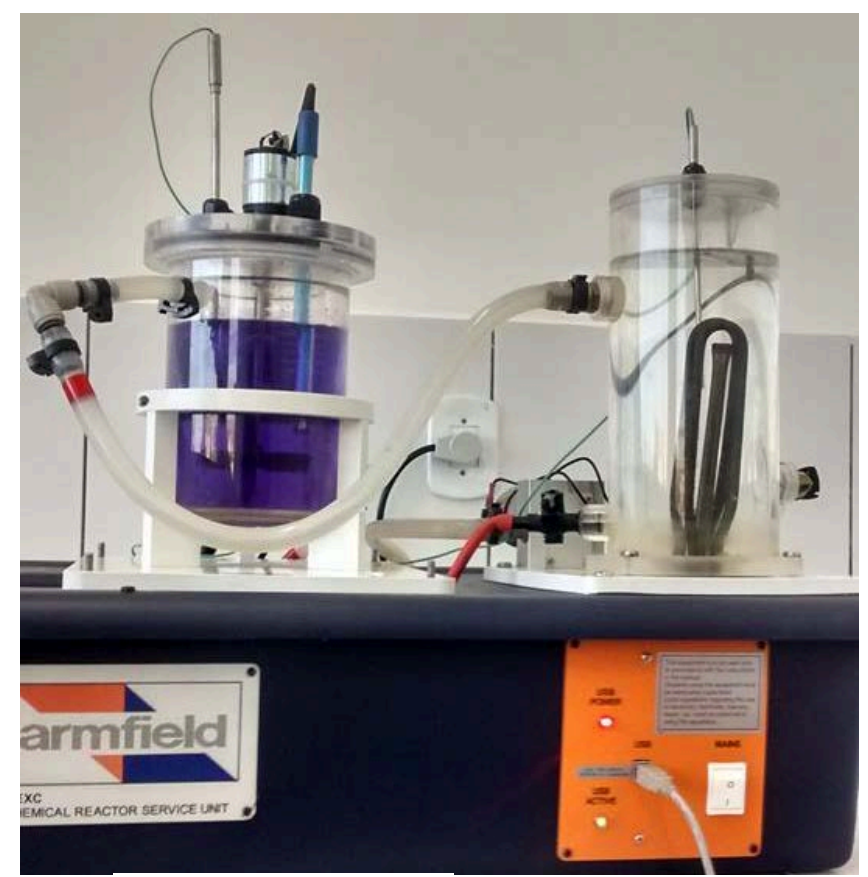

Fonte: Elaborada pelos autores.

Tabela1 - Condições das reações realizadas no experimento a $\mathrm{T}_{\text {reator }}=30^{\circ} \mathrm{C}$

\begin{tabular}{ccc}
\hline Reação & Hidróxido de Sódio $\left(\mathbf{m o l} / \mathbf{d m}^{3}\right)$ & Acetato de Etila $\left(\mathbf{m o l} / \mathbf{d m}^{3}\right)$ \\
\hline 1 & 0,1 & 0,1 \\
2 & 0,1 & 0,08 \\
3 & 0,1 & 0,04 \\
\hline
\end{tabular}

Fonte: Elabora pelos autores.

Os dados foram coletados pelo software CEB - Armfield, desenvolvido pelos fabricantes do reator, armazenados e exportados para uma planilha no Excel®.

\section{Resultados e discussão}

Com os dados coletados traçou-se a curva que relaciona a concentração final de $\mathrm{NaOH}$ com o tempo de reação (Gráfico 1). A concentração final de $\mathrm{NaOH}$ foi calcula pela Equação 2.

$$
X_{A}=\frac{C_{A 0}-C_{A}}{C_{A 0}}
$$

Onde $\mathrm{C}_{\mathrm{A} 0}$ e $\mathrm{C}_{\mathrm{A}}$ são as concentrações inicial e final do Hidróxido de Sódio, respectivamente, e $\mathrm{X}_{\mathrm{A}}$ é o valor da sua conversão. 
Gráfico 1 - Gráfico da concentração NaOH em função do tempo, com a concentração de Acetato de Etila igual a $0,1 \mathrm{~mol} / \mathrm{dm}^{3}$.

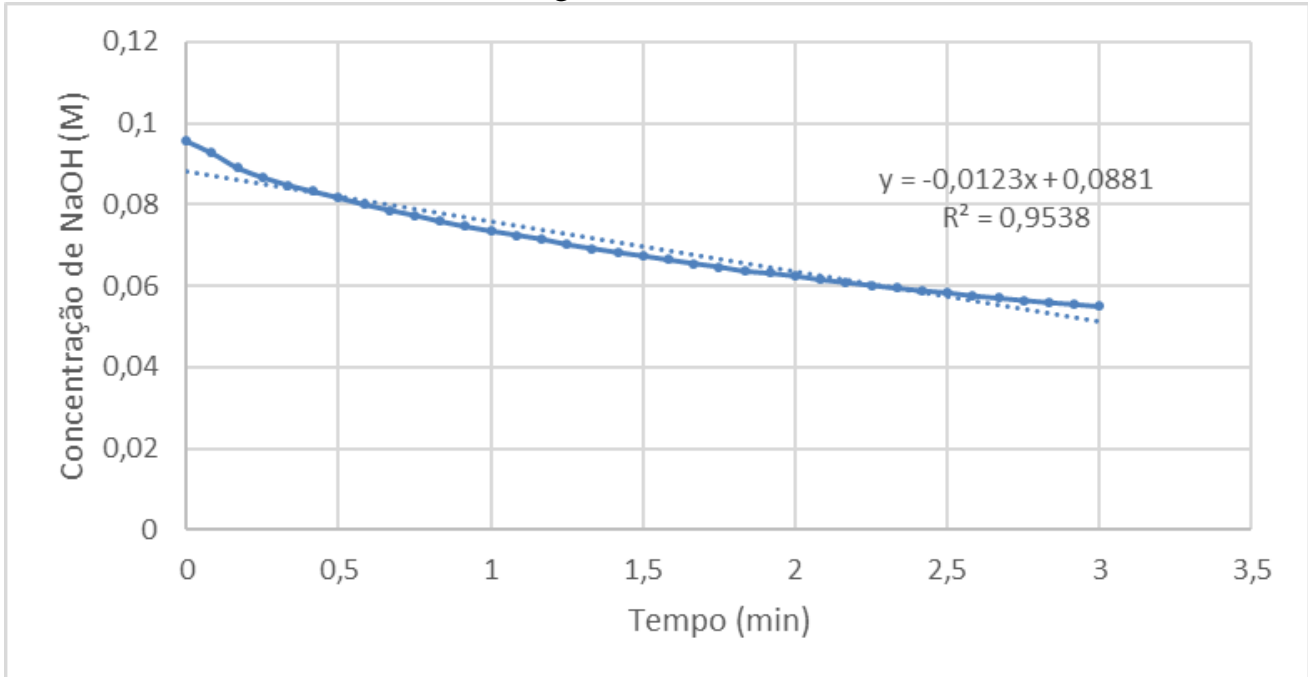

Fonte: Elaborada pelos autores.

Em seguida, para determinar a velocidade de reação inicial, calculou-se a derivada da curva em análise no tempo inicial ( $\mathrm{t}=0$ ) dada pela Equação 3.

$$
\frac{d C_{A 0}}{d t}=\left(-r_{A}\right)
$$

O mesmo procedimento de cálculo foi repetido para os outros valores de concentração de Acetato de Etila e as curvas analisadas são mostradas nos Gráficos 2 e 3.

Gráfico 2 - Gráfico da concentração $\mathrm{NaOH}$ em função do tempo, com a concentração de Acetato de Etila igual a $0,04 \mathrm{~mol} / \mathrm{dm}^{3}$.

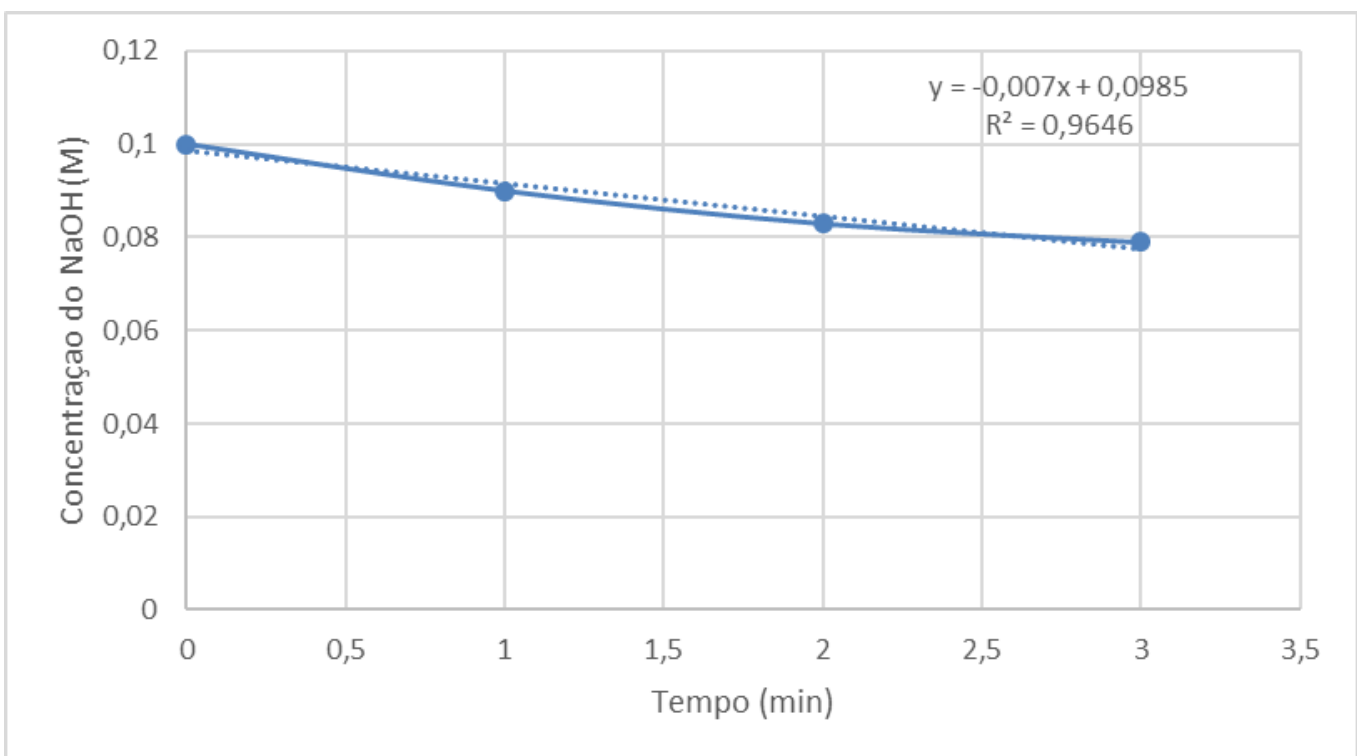

Fonte: Elaborada pelos autores. 
Gráfico 3 - Gráfico da concentração NaOH em função do tempo, com a concentração de Acetato de Etila igual a $0,08 \mathrm{~mol} / \mathrm{dm}^{3}$.

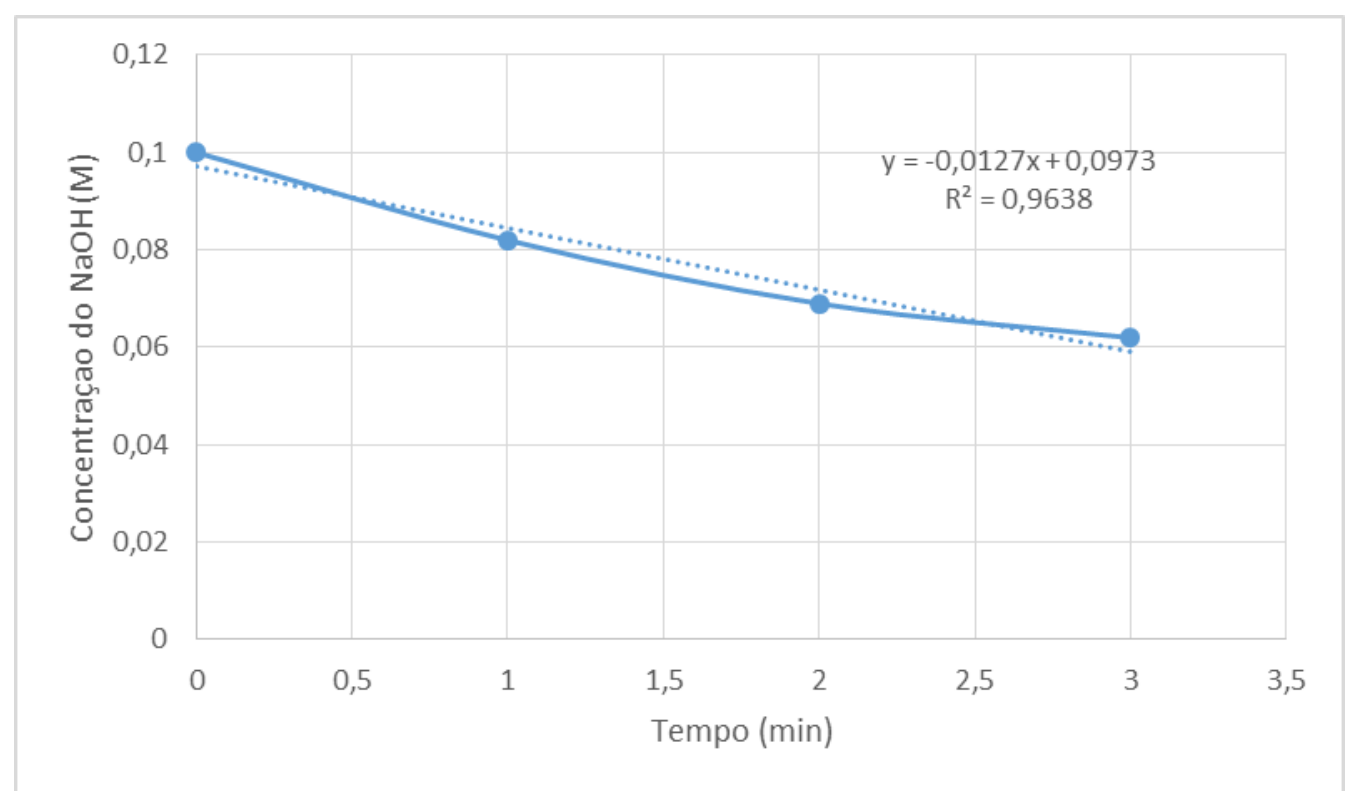

Fonte: Elaborada pelos autores.

Para determinar a ordem da reação, considerou-se algumas afirmações de acordo com as relações a seguir:

$$
\begin{gathered}
\left(-r_{A}\right)=k C_{A}^{n} C_{B}^{m} \\
\left(-r_{A, 0}\right)=k C_{A 0}^{n} C_{B 0}^{m} \\
\left(-r_{A, 0}\right)=k^{\prime} C_{B 0}^{m}
\end{gathered}
$$

Onde, $k^{t}=k C_{A 0}^{n}$ e B é o índice que representa o Acetato de Etila.

Logo,

$$
\ln \left(-r_{A, 0}\right)=\ln k^{\prime}+m \ln \left(C_{B 0}\right)
$$

Com base nos resultados obtidos anteriormente, tem-se a Tabela 2:

Tabela 2 - Resultados obtidos.

\begin{tabular}{ccccc}
\hline Reação & $\left(-\boldsymbol{r}_{\boldsymbol{A}, \mathbf{0}}\right)$ & $\ln \left(-\boldsymbol{r}_{\boldsymbol{A}, \mathbf{0}}\right)$ & $\boldsymbol{C}_{\boldsymbol{B} \mathbf{0}}^{m}$ & $\ln \left(\boldsymbol{C}_{\boldsymbol{B} \mathbf{0}}\right)$ \\
\hline $\mathbf{1}$ & 0,0123 & $-4,39815602$ & 0,1 & $-2,30258509$ \\
$\mathbf{2}$ & 0,0070 & $-4,96184513$ & 0,04 & $-3,21887582$ \\
$\mathbf{3}$ & 0,0127 & $-4,36615328$ & 0,08 & $-2,52572864$ \\
\hline
\end{tabular}

Fonte: Elaborada pelos autores.

Posteriormente, plotou-se o gráfico de $\ln \left(-r_{A_{0} 0}\right)$ em função de $\ln \left(C_{B 0}\right)$, para determinação da ordem da reação para o Acetato de Etila e encontrou-se um valor aproximado de 0,673, coerente com a literatura proposta por KRÜGER et al. (2013), que determina uma faixa para a reação em questão entre 0 e 2.

Nos Gráficos 1, 2 e 3 é importante destacar que a não linearidade dos dados experimentais torna a regressão linear apenas uma aproximação do parâmetro. 
Quanto à conversão de $\mathrm{NaOH}$, verifica-se que no experimento de concentração de 0,1M para o Acetato de Etila a conversão foi maior quando comparado com os outros experimentos, com concentração de Acetato de Etila de 0,04M e 0,08M. Esse fato se dá em virtude de não existir reagente limitante, visto que os coeficientes estequiométricos dos reagentes são iguais. Quando as proporções entre os reagentes são diferentes, pode ocorrer de parte de um deles não reagir efetivamente e provocar quedas nos valores de conversões.

\section{Conclusão}

Os resultados das medidas para a determinação da velocidade da reação em estudo mostram que as taxas de variação dependem da concentração dos reagentes. Sendo assim, é possível representar essa dependência em termos de equações diferenciais que representam a lei de velocidade. As soluções dessas equações são utilizadas para prever as concentrações de espécies, em qualquer momento após o início da reação. A forma da lei de velocidade também fornece certa compreensão sobre a série de passos elementares através dos quais a reação ocorre. Nesse contexto, pode-se concluir que o experimento apresentou uma metodologia eficiente na determinação da ordem de reação da saponificação do Acetato de Etila. Entretanto, como foi possível observar, os coeficientes de determinação não resultaram em valores elevados, mas foi coerente, visto que a ordem da reação esteve na faixa determinada pela literatura de KRÜGER et al., 2013.

\section{Agradecimento}

Os autores desse trabalho agradecem a Universidade Federal de Campina Grande e à Unidade Acadêmica de Engenharia Química.

\section{Collection and analysis of kinetic data of Ethyl Acetate the saponification reaction in a busy Batch Reactor}

Albstract: The Ethyl acetate is a family organic compound of esters with high solvency power widely used in industrial paints and varnishes, but can also be used on substrates such as cellulosic film, paper, aluminum foil, PVC, polyester and polyethylene polypropylene. When this compound is reacted with a strong base, it is hydrolysed and this reaction is called saponification. The reaction is complex and not simple with several intermediate reactions, so it is necessary to study and know the kinetic methods which consist of analyzes of correlations based on kinetic data from experimental procedures. In this work we applied the differential method with the aim of finding a reaction order to satisfy the reaction conditions of the process. Three concentrations were used for Etlia acetate $(0.1 \mathrm{M}$, $0.04 \mathrm{M}$ and $0.08 \mathrm{M}$ ) and the data collected from transient. The final concentration of the base used as reagent (Sodium Hydroxide) can be measured from conversion values calculated by the CEB-Armfield software. Based on these data, charts the concentration of sodium hydroxide versus time were plotted and the derivatives of the curves analyzes were calculated by linear regression enabling the calculation of the reaction order, however, it is important to note that the nonlinearity of the experimental data becomes linear regression only an approximation parameter. As regards the $\mathrm{NaOH}$ conversion verifies that the $0.1 \mathrm{M}$ concentrations for the experiment Ethyl acetate conversion was higher when compared with the other experiments. This fact occurs because there is no limiting reagent, causing the reactants to react effectively causing an increase in the conversion value.

Keywords: Ethyl acetate; CEB-Armfield; order reaction; conversion 


\section{Referências bibliográficas}

CHANG, Raymond. Química Geral: Conceitos essenciais, 4ª ed. Editora AMGH. Porto Alegre: 2010.

CHEMISOL - Acessado em 27 de Setembro de 2015. Disponível em. http://www.chemisol.com.br/produtos/acetato-de-etila/

FOGLER, H. Scott. Elementos de Engenharia das Reações Químicas, 4ª ed. Editora LTC. São Paulo: 2009.

FROST, Arthur Atwater; PEARSON, G. Ralph. Kinetics and Mechanism: A Study of homogeneous Chemical Reactions, $1^{\text {a }}$ ed. Editora Wiley. Nova Iorque: 1952.

HILL, Charles G.; ROOT, Thatcher W. Introduction to Chemical Engineering Kinetics \& Reactor Design, $2^{\text {a }}$ ed. Editora Wiley. Nova Iorque: 1977.

LEVENSPIEL, Octave. Engenharia das reações Químicas, $3^{\text {a }}$ ed. Editora Blucher. São Paulo: 2000.

KRÜGER, Adolfo Guilerme; REIS, Camila dos Santos; POSTAL, Kahoana. Cinética da saponificação do Acetato de Etila - Determinação da constate de velocidade da reação, Universidade Federal do Paraná. Curitiba: 2013.

SOLOMONS, T. W. Graham; FRYHLE, Craig. B. Organic Chemistry, 9a ed. Editora Wiley. Nova Iorque: 2008. 